\title{
Tests of phenotypic and genetic concordance and their application to the conservation of Panamanian golden frogs (Anura, Bufonidae)
}

\author{
CORINNE L. RICHARDS and L. LACEY KNOWLES \\ Department of Ecology and Evolutionary Biology, University of Michigan, Museum of Zoology, 1109 Geddes Avenue, Ann Arbor, \\ MI 48109-1079, USA
}

\begin{abstract}
Evolutionarily significant units (ESUs) differ in the extent to which they capture, or even consider, adaptive variation, and most such designations are based solely on neutral genetic differences that may not capture variation relevant to species' adaptabilities to changing environmental conditions. While concordant patterns of divergence among data sets (i.e. neutral and potentially non-neutral characters) can strengthen ESU designations, determining whether such criteria are met for highly variable taxa is especially challenging. This study tests whether previously defined ESUs for endangered Panamanian golden frogs (Atelopus varius and Atelopus zeteki) exhibit concordant variation among multiple phenotypic traits and mitochondrial DNA sequences, and the extent to which such divergence corresponds to environmental differences. Multivariate analyses identify phenotypic and genetic differentiation consistent with proposed ESUs and support the status of $A$. varius and $A$. zeteki as separate species. Moreover, the significant association detected between ESU co-membership and genetic similarity, which remained strong after removing the effect of geographic distance, also indicates that genetic differences are not simply due to isolation by distance. Two phenotypic characters (body size and the extent of dorsal black patterning) that differ among ESUs also co-vary with environmental differences, suggesting that to the extent that these phenotypic differences are heritable, variation may be associated with adaptive divergence. Lastly, discriminant function analyses show that the frogs can be correctly assigned to ESUs based on simultaneous analysis of multiple characters. The study confirms the merit of conserving the previously proposed golden frog ESUs as well as demonstrates the utility and feasibility of combined analyses of ecological, morphological and genetic variation in evaluating ESUs, especially for highly variable taxa.

Keywords: Atelopus, character concordance, evolutionarily significant units (ESUs), genetic variation, morphological variation, $\mathrm{mtDNA}$
\end{abstract}

Received 10 January 2007; revision accepted 4 April 2007

\section{Introduction}

In the endeavour to protect biological diversity, the field of conservation biology also contends with the challenge of preserving the functionality of evolutionary processes necessary for sustaining it in the face of anthropogenic changes (Moritz 2002; Monsen \& Blouin 2003). Defining evolutionarily significant units (ESUs) - the intraspecific units meriting separate conservation (Ryder 1986) - is

Correspondence: Corinne L. Richards, Fax: 734-763-4080; E-mail: clrichar@umich.edu central to achieving these goals. However, the identification of ESUs is far from straightforward and has been the subject of much debate (Avise 1989; Moritz 1994; Grant 1995; Bowen 1999; Dimmick et al. 1999; Paetkau 1999; Taylor \& Dizon 1999; Crandall et al. 2000; Green 2005). Consequently, the extent to which such units consider adaptive variation (Waples 1991; Bowen 1999; Crandall et al. 2000) in addition to patterns of neutral-genetic variation (Avise 1989; Moritz 1994; Dimmick et al. 1999) differs substantially.

Independent characters, such as neutral genetic haplotypes and heritable phenotypes (e.g. Rising \& Avise 1993; 
Ryan \& Bloomer 1999; Friesen et al. 2006; Gharrett et al. 2006; Gompert et al. 2006) each provide a separate historical record of population divergence and are expected to show concordant variation if they share a history of divergence (Ryder 1986; Avise 1989; Grady \& Quattro 1999). Analysis of such concordant variation provides an evolutionary framework that affords insight into the processes shaping variation and is therefore instrumental in developing conservation strategies to not only sustain viable populations but also to maintain the context for selection in the face of a changing environment (Ryder 1986; Avise 1989; Dizon et al. 1992; Vogler et al. 1993; Paetkau 1999; Crandall et al. 2000; Moritz 2002). Irrespective of whether preserving adaptive variation is a significant consideration for a particular conservation initiative, ESU designations based on multiple characters reduce the risk of being inaccurate. For example, ESUs diagnosed using the single criterion of genetic distinctiveness (which is often based on analyses of mitochondrial DNA) may not be accurate, as recent divergence (Lorenzen et al. 2006), large effective population sizes (Paetkau 1999), or introgression (Gompert et al. 2006) can slow the formation of genetic structure and therefore bias conservation assessments. Likewise, evolutionary relationships and divergence arising from historical processes may not be represented if ESUs are diagnosed solely from phenotypic characters subject to intense selection or that exhibit nonheritable variation (Avise 1989; Grady \& Quattro 1999). Additionally, multivariate analyses that reveal concordance among multiple character sets reduce the potential for taxonomic ambiguities, which arise more often when decisions are based on single characters (Avise 1989; Grady \& Quattro 1999; Paetkau 1999).

Tests of concordance across multiple independent characters are especially important for highly variable species with poorly understood taxonomy (e.g. ciscoes: Turgeon \& Bernatchez 2003; Galapagos petrels: Friesen et al. 2006; and long-billed larks: Ryan \& Bloomer 1999). When faced with this situation for endangered species, the additional urgency emphasizes the importance of designating ESUs that capture variation essential to population persistence as species have to contend with a variety of challenges, ranging from habitat loss and fragmentation to climate change and disease. This study uses multivariate analyses of character concordance to evaluate the adequacy of ESUs previously proposed for conserving the Panamanian golden frogs (Atelopus varius and Atelopus zeteki). These frogs show a great deal of morphological, ecological, genetic, and demographic variation among geographically proximate populations in western Panama. The species are also critically endangered (IUCN 2004) due to habitat loss and fragmentation, illegal collection for the pet trade, and chytridiomycosis, a fungal epizootic (Zippel 2002). Patterns of phenotypic and genetic differences among golden frog populations are examined in a quantitative framework, using both single and multivariate analyses, to assess the level of concordance among characters as well as the level of support for five previously proposed ESUs (Zippel et al. 2007) based on qualitative descriptors of phenotypic variation (Fig. 1).

In contrast to examples in which species are phenotypically homogeneous but genetically differentiated (jellyfish: Holland et al. 2004; king weakfish: Santos et al. 2006), or phenotypically diverse yet genetically undifferentiated (hamlets: McCartney et al. 2003; poison dart frogs: Summers et al. 1997; sticklebacks: Taylor \& McPhail 1999), the genetic and phenotypic divergence among Panamanian golden frogs is concordant, with populations exhibiting significant differentiation in both. However, the magnitude of divergence differs among phenotypic traits and with the amount of genetic differentiation. This study highlights the profound effect that taxonomy, character choice and the choice of a single vs. multiple character approach can have on the interpretation of patterns of variation and their implications for developing conservation priorities. In addition to contributing to our understanding of the processes underlying variation in golden frogs relevant to their conservation, this study demonstrates the general applicability of analyses of molecular variance (AMOvAs) and Mantel tests in (i) identifying ESUs based on the level of concordance among genetic and phenotypic variation, and (ii) assessing support for alternative evolutionary and ecological mechanisms in the generation of phenotypic variation.

\section{Materials and methods}

Toe-clip tissue samples, photographs, and snout-vent lengths (SVLs) were collected for Atelopus varius and Atelopus zeteki from populations throughout their extant ranges in Panama (Fig. 1); this sampling included 2-4 populations per ESU. All animals were captured by hand and released at the point of capture within $5 \mathrm{~min}$ of data collection. While A. varius was historically found throughout much of montane Costa Rica and Panama, by the time of this study its extant range had been reduced to the area sampled here.

\section{Genetic data}

Tissues were preserved in a salt-saturated DMSO and EDTA solution. A total of 141 individuals were sequenced including frogs from 15 populations (Fig. 1). Four outgroup specimens were also used: three $A$. varius from Monteverde, Costa Rica (MVZ Herp 149729 and 164816-7) and one Atelopus senex from near Volcan Barba, Costa Rica (MVZ Herp 149735). Due to the huge morphological variability seen among Atelopus populations in Central America and the lack of a phylogeny for the group, the taxonomy of 


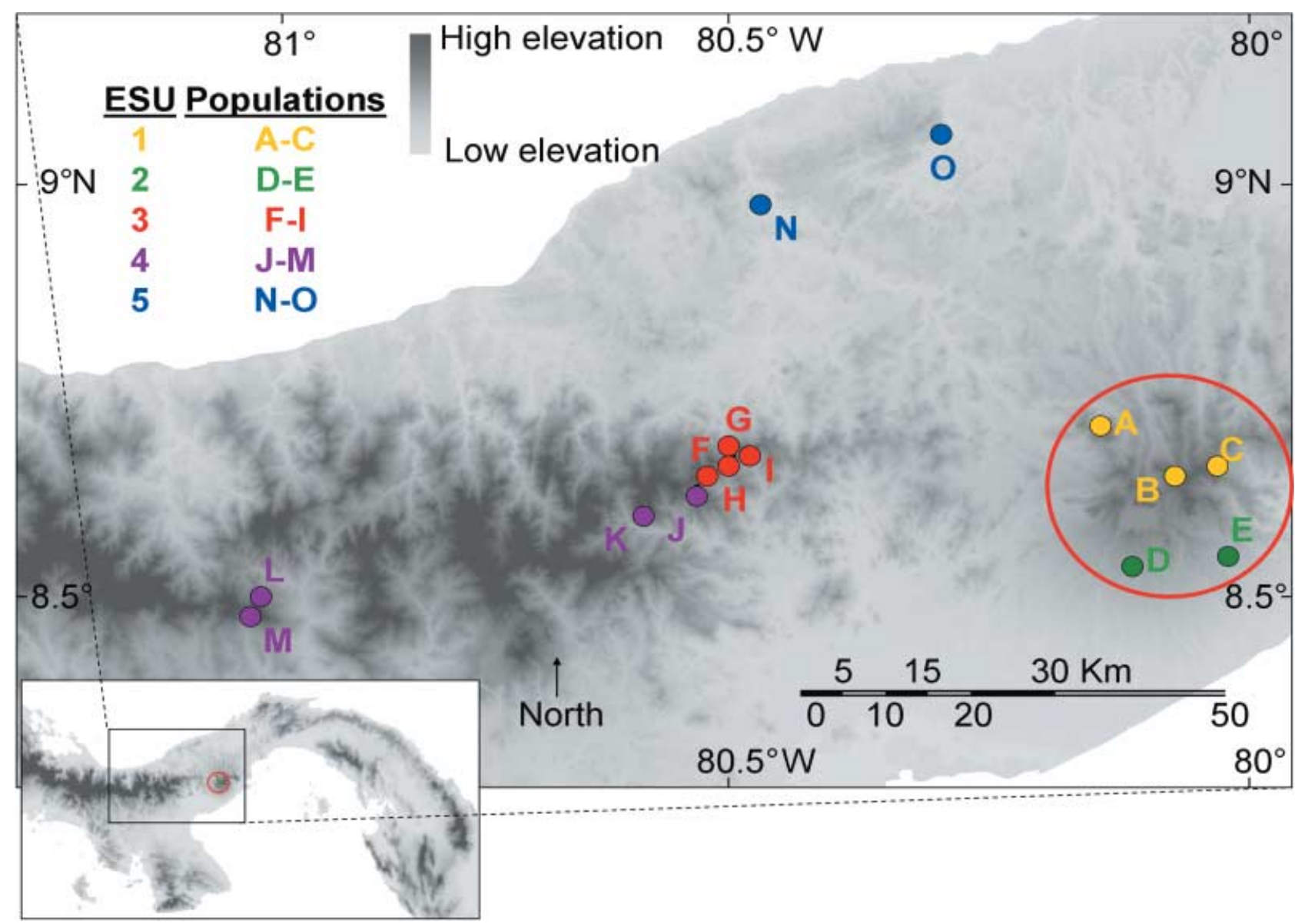

Fig. 1 Map of Panama (lower left) and enlargement (upper right) showing the approximate locations of populations sampled across the extant range of the golden frogs and proposed ESU membership (following Zippel et al. 2007). The red circle encloses the area of the El Valle crater, the putative range of Atelopus zeteki. Populations outside of the circle are within the historical range of Atelopus varius (Savage 1972).

these species is not well understood. The $A$. varius samples from Monteverde were included in the outgroup as they were 5-6\% divergent from any of the ingroup haplotypes and are likely to represent a distinct species. This population is as divergent from Panamanian A. varius as are populations of $A$. senex from Costa Rica, and in fact, the Monteverde $A$. varius samples are only $0.5-1.2 \%$ divergent from $A$. senex, indicating taxonomic confusion for these records. Genomic DNA was isolated using a QIAGEN DNeasy kit according to the standard protocol for animal tissue and $10 \mu \mathrm{L}$ of extracted DNA was added to $20 \mu \mathrm{L}$ of GeneReleaser (BioVentures) according to the general protocol to remove polymerase chain reaction (PCR) inhibitors. A 755-base pair fragment of cytochrome $b$ (cytb) and 630 base pairs of cytochrome oxidase I (COI) were sequenced from the mitochondrial genome using primers COIfXenL and COIfXenH (see Palumbi et al. 1991) for COI and MVZ25-L (see Moritz et al. 1992) and ControlW$\mathrm{H}$ (see Goebel et al. 1999) for cytb. Twenty-five microlitre polymerase chain reactions with an initial denaturing at $94^{\circ} \mathrm{C}$ for $2 \mathrm{~min}$ followed by 35 cycles of $30 \mathrm{~s}$ denaturing at $94{ }^{\circ} \mathrm{C}, 45 \mathrm{~s}$ annealing at either $60^{\circ} \mathrm{C}$ for cyt $b$ or $45^{\circ} \mathrm{C}$ for COI, and $30 \mathrm{~s}$ extension at $72^{\circ} \mathrm{C}$ were used. PCR products were cleaned with a QIAquick kit prior to automated sequencing.

\section{Phenotypic and environmental data}

Digital photographs of the dorsal and ventral surfaces, as well as body size (SVL) measurements were taken in the field for 213 adult male frogs from the same 15 populations mentioned above (Fig. 1). Only adult males were measured because body size is a sexually dimorphic trait in this species. To quantify individual colour variation, the colour and contrast values among digital photographs were standardized by adjusting midtones to a target value using the 'auto colour' command in Adobe Photoshop CS version 8.0. Yellow and black colours were characterized with Image-Pro Discovery version 4.5 (Media Cybernetics) by averaging the RGB (red, green, blue) colour values from 
three random points on the frog's dorsal surface. Individual variation was summarized with a principal components analysis (PCA). PCAs of averaged R, G, and B values extracted single significant components and for both yellow and black colours and the R, G, and B values all contributed significantly to the $\mathrm{z}$-scores $(\mathrm{z}$-yellow $=0.744 \mathrm{R}$ $+0.896 \mathrm{G}+0.807 \mathrm{~B}$ and $\mathrm{z}$-black $=0.890 \mathrm{R}+0.983 \mathrm{G}+0.881 \mathrm{~B})$. The $z$-scores for the significant components of yellow and black dorsal colours and two other phenotypic traits the percentage of black patterning on the dorsal surface of each frog (as quantified with Image-Pro Discovery) and the SVL (as measured in the field with dial calipers) - were analysed in both univariate and multivariate frameworks (see below). Colour and pattern variables as well as body size were chosen for analysis because they are highly variable among populations and potentially adaptive. Because golden frogs are highly toxic, their black and yellow colouration is likely aposematic and an adaptation to avoid predation. Body size differences may also represent adaptations to variation in climate throughout their range.

Altitude and locality data for each population was measured using a hand-held GPS unit. Climate data were extracted for each locality with the program DIVAGIS (version 5.2: www.diva-gis.org), using values from WorldClim interpolated climate layers (approximately 1$\mathrm{km}$ spatial resolution). These layers were developed from monthly climate measurements compiled from weather stations around the globe for the years 1950-2000 (Hijmans et al. 2005). We used the program to extract the climate values for each locality given its geographic coordinates. Nineteen bioclimatic variables reflecting annual trends in temperature and precipitation, seasonality, and extreme environmental factors (e.g. temperature of the coldest month) were used (see www.worldclim.org/bioclim.htm for detailed descriptions of individual variables). Variation in the bioclimatic variables and altitude among populations was summarized using PCA, resulting in three composite climate variables (hereafter referred to as bioclim 1-3, Table 1).

\section{Statistical analyses}

Because of taxonomic uncertainty (see below), all statistical analyses were conducted treating the putative species, $A$. varius and $A$. zeteki, separately, as well as considering them as a single taxon. Pronounced variability within $A$. zeteki and $A$. varius and problems with the specimens and characters used historically to diagnose the two species raise uncertainty about their taxonomic status. The original description of $A$. zeteki as a subspecies of $A$. varius was based on differences in colouration, colour patterning, and body size of frogs from El Valle, Panama (Dunn 1933). However, this designation was not based on a comparison with Panamanian A. varius, but instead Atelopus cruciger from Venezuela and a specimen of unknown taxonomy from Colombia (Lotters 1996). Differences in skin toxins (Villa et al. 1988) and male calls (Cocroft et al. 1990) were later used to elevate $A$. zeteki from a subspecies to a species. However, subsequent studies have shown that the toxin which was supposedly unique to $A$. zeteki is also found in A. varius (Yotsu-Yamashita et al. 2004). Furthermore, the bio-acoustic differences of Cocroft et al. (1990) actually represent intraspecific differences in $A$. varius calls as their 'A. zeteki' specimen was in fact from a population of

\begin{tabular}{lrrr}
\hline Original variable & Bioclim 1 & Bioclim 2 & Bioclim 3 \\
\hline Mean annual temperature & 0.935 & 0.224 & -0.265 \\
Monthly temperature range & -0.892 & 0.072 & -0.081 \\
Isothermality & -0.739 & 0.417 & -0.153 \\
Temperature seasonality & 0.873 & 0.285 & 0.258 \\
Maximum temperature of warmest month & 0.881 & 0.320 & -0.295 \\
Minimum temperature of coolest month & 0.947 & 0.242 & -0.206 \\
Annual temperature range & -0.841 & 0.012 & -0.063 \\
Average temperature of wettest quarter & 0.915 & 0.252 & -0.300 \\
Average temperature of driest quarter & 0.919 & 0.230 & -0.312 \\
Average temperature of warmest quarter & 0.940 & 0.240 & -0.232 \\
Average temperature of coolest quarter & 0.922 & 0.245 & -0.285 \\
Mean annual precipitation & 0.824 & -0.264 & 0.487 \\
Precipitation of wettest month & 0.220 & 0.832 & 0.483 \\
Precipitation of driest month & 0.595 & -0.792 & 0.096 \\
Precipitation seasonality & -0.604 & 0.783 & -0.114 \\
Precipitation of wettest quarter & 0.442 & 0.548 & 0.685 \\
Precipitation of driest quarter & 0.667 & -0.718 & 0.164 \\
Precipitation of warmest quarter & 0.851 & -0.485 & 0.159 \\
Precipitation of coolest quarter & 0.751 & 0.332 & 0.466 \\
Altitude & -0.913 & 0.112 & 0.272 \\
\hline
\end{tabular}

Table 1 Component matrix for climate PCA. Absolute values of component loadings indicate correlations between components (bioclim 1, 2 and 3) and the original variables. Bioclim 1 is strongly influenced by measures of temperature whereas bioclim 2 and 3 are more strongly influenced by precipitation. 
A. varius (Zippel et al. 2007). Further studies are needed to determine whether the two nominal species can be differentiated by call.

Genetic data. Phylogenetic trees were estimated using maximum parsimony (MP), maximum likelihood (ML) and Bayesian inference with the sequence data from the two mitochondrial genes concatenated into a single haplotype for each individual (GenBank Accession numbers for cyt $b$ and COI: EF494908-EF494999). MP and ML analyses were performed in PAUP $4.0 \mathrm{~b} 10$ (Swofford 2002) using 500 random-stepwise heuristic searches with tree-bisection-reconnection branch-swapping and branch support was assessed using 1000 and 100 nonparametric bootstrap replicates for MP and ML, respectively. An HKY $+\Gamma$ model (Hasegawa et al. 1985) was selected as the best fit model $\left(\pi_{\mathrm{A}}=0.26842, \pi_{\mathrm{C}}=0.24303, \pi_{\mathrm{G}}=0.14292\right.$, $\pi_{\mathrm{T}}=0.34564$, t-ratio $=6.624, \alpha=0.2226$, rate categories $=5$ ) using DT-MODSEL (Minin et al. 2003) and used for ML and Bayesian analyses. Four chains of $10^{7}$ generations with trees saved every $10^{3}$ generations were used for the Bayesian analysis after a burn in of $10^{4}$ generations (MRBAYES 3.0b4: Huelsenbeck \& Ronquist 2001). Net sequence divergence (Nei 1987) was estimated using DNASP version 4.10.

AMOVAs with individuals nested by population and the proposed ESUs were performed in ARLEQUIN 2.0 (Excoffier et al. 1992; Shchneider et al. 2000) using 1000 permutations, and a Kimura 2-parameter distance matrix with $\Gamma=0.2226$. Mantel tests were used to test for correlations between (i) log-transformed genetic $\left(F_{\mathrm{ST}}\right.$ values, the dependent variable, or DV) and straight-line geographic distances (the independent variable, or IV) (Slatkin 1993), and (ii) log-transformed genetic distances (DV) and an indicator matrix $(0,1)(\mathrm{IV})$ that designates populations as members of the same (0), or different (1) ESUs. These tests were performed following the procedure of Legendre \& Legendre (1998) in IBD version 1.5 (Bohonak 2002) with $10^{4}$ randomizations. Partial-Mantel tests were used to assess correlations between log-transformed genetic (DV) and geographic distances (IV) while controlling for ESU membership (DV), and log-transformed genetic distance (DV) and ESU membership (DV) while controlling for geographic distance (IV).

Phenotypic variation. To test whether and how individuals from the five proposed ESUs and two putative species differ in phenotype, individual variables encompassing four morphological aspects of phenotype were analysed separately, as well as jointly. As variances were highly unequal across ESUs and species, Kruskal-Wallis tests (nonparametric alternative to ANOvAs) were used to compare means and nonparametric multiple comparison tests (Dunnett's C) were used to determine which groups differed significantly for a particular phenotypic character.
Multivariate discriminant function analyses (DFAs) were used to determine the extent to which individual frogs can be differentiated according to ESU (we did not use DFAs to test for statistical significance of phenotypic differences among ESUs because the covariance matrices were unequal and this could affect $P$ values, see Manly 2005). Cross-validation of ESU classifications was accomplished by classifying each individual by the functions derived from all others (leave-one-out classification) and prior probabilities for each ESU were set proportionally.

To assess the relative support for alternative causes of variation among golden frog populations, pairwise Mantel tests were performed among genetic, geographic, morphological, and environmental variables. Correlations between (i) morphological (DV) and environmental (IV) phenotypic variables, and (ii) phenotypic variables (DV) and genetic distance (pairwise $F_{\mathrm{ST}}$ values, IV) were investigated using Mantel tests in the Microsoft Excel 2000 PopTools add-in (Hood 2000). Because pairwise Mantel tests were used to screen for potential correlations rather than test specific hypotheses about the relationships between pairs of variables, significance levels were not adjusted for multiple hypothesis testing (see Nakagawa 2004 and Roback \& Askins 2005). Least-square linear regressions (run in sPss, version 14.0) were then used to test for correlations among pairs of variables with significant Mantel tests and generate hypotheses about their causes that could be tested in the future.

\section{Results}

\section{Genetic variation}

Two strongly supported clades (Fig. 2) corresponding generally to populations of Atelopus varius and Atelopus zeteki were recovered in each analysis $\mathrm{MP}, \mathrm{ML}$ and Bayesian). For the MP analysis, 126 of the 1385 characters were parsimony informative and 304 equally parsimonious trees of 213 steps were found. The two clades are separated by $3.32 \%$ net sequence divergence (Nei 1987, equation 10.20). All but three populations were restricted to one of the two clades - individuals from one A. zeteki population (A) and two A. varius populations ( $\mathrm{G}$ and $\mathrm{N}$ ) are distributed across both clades, although haplotypes from the El Valle clade were rare as seven of the 43 individuals from these populations carried haplotypes from the El Valle clade (Figs 1 and 2). Repeated DNA extraction and resequencing confirmed that these populations were indeed composed of a mixture of haplotypes from the two clades. Sixty unique mitochondrial haplotypes, 49 of which were population-specific, resulted from the 141 individuals sequenced, excluding outgroups (Table 2).

Genetic structure was also revealed by the $F_{\mathrm{ST}}$ analyses, with significant $F_{\mathrm{ST}}$ values observed among the majority of 


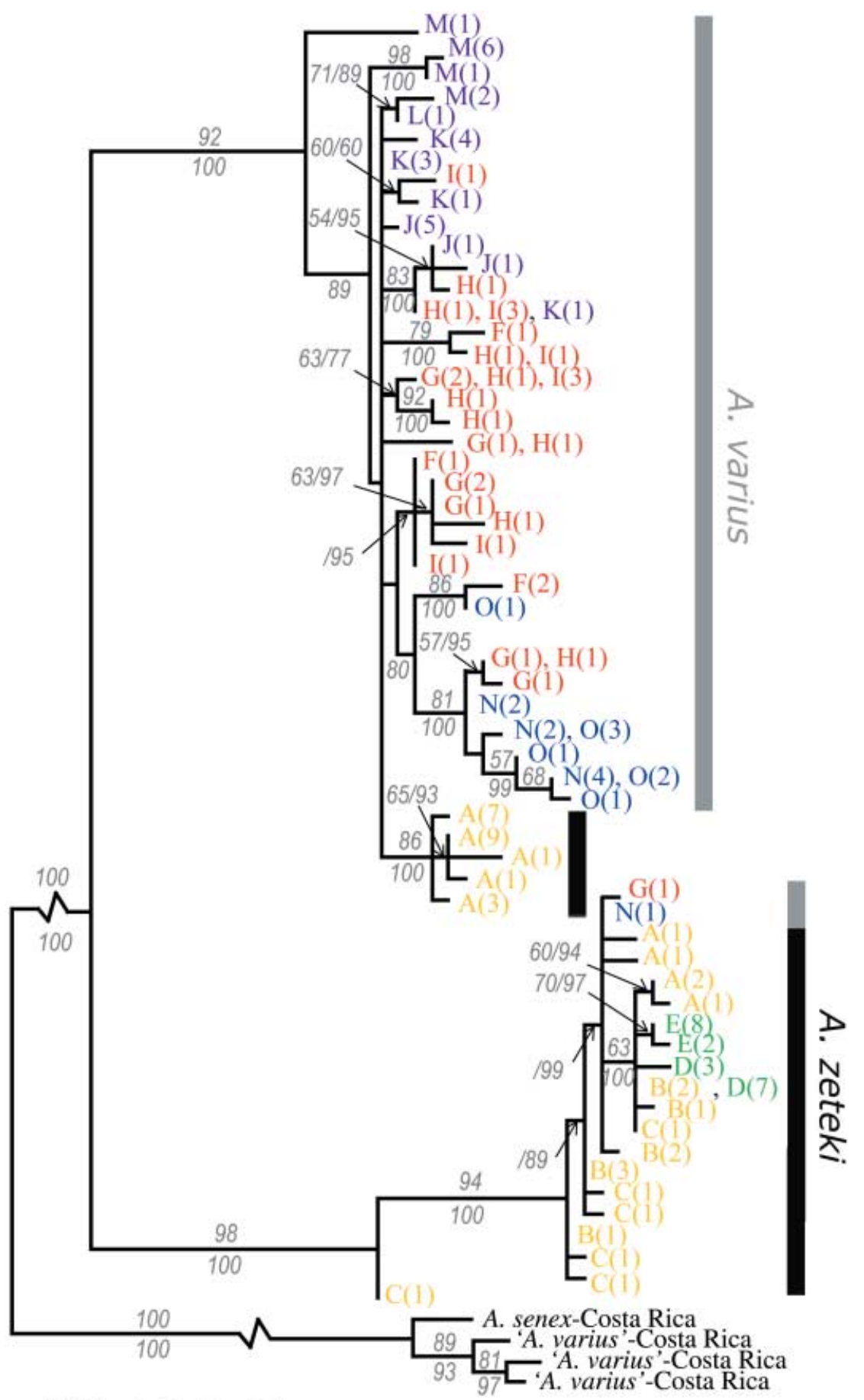

Fig. 2 ML tree showing the two clades recovered in all phylogenetic analyses. Haplotypes are colour-coded by ESU and labelled by population (according to the colour scheme and letter designations in Fig. 1). The number of individuals with a particular haplotype is given in parentheses; grey and black bars indicate Atelopus varius and Atelopus zeteki haplotypes, respectively. ML bootstraps are given first followed by Bayesian support values, or are shown above and below the branch, respectively (scores of 50 or less are not reported). Parsimony bootstrap scores (not shown) exceeded ML and Bayesian support values in every case. The likelihood of this tree is 3196.19. The haplotypes labelled as ' $A$. varius' in the outgroup are from Monteverde, Costa Rica, and are more similar to Atelopus senex than any of the ingroup haplotypes suggesting taxonomic confusion for these outgroup samples.

\section{-0.001 substitutions/site}

pairwise population comparisons (Table 2), including geographically proximate ones. For example, $F_{\mathrm{ST}}$ values were significant between populations $\mathrm{F}$ and $\mathrm{J}$ despite being separated by only $1.5 \mathrm{~km}$ (Fig. 1). The amovas showed significant hierarchical structuring of genetic variation as well (Table 3). When considered as higher groupings, both ESUs and species (A. varius vs. A. zeteki) explained a significant partitioning of genetic variance among popula- tions (Tables 3a, b). However, the effect of ESU groupings on genetic structure was not consistently significant when the species were analysed separately - ESU groupings explained a significant amount of genetic variance in A. varius (Table 3c) but not A. zeteki (Table 3d).

Genetic and geographic distances were correlated in each species (A. varius Mantel: $r=0.47, P=0.002$; A. zeteki Mantel: $r=0.817, P=0.04)$. This pattern of isolation by 
Table 2 Distribution of mtDNA haplotypes and genetic structure among populations (letters correspond to localities on Fig. 1). Number of individuals, $N$; number of haplotypes, $h$, number of haplotypes exclusive to that population, (excl.); and pairwise $F_{\mathrm{ST}}$ values are given to the right of the dashed line. Boxes enclose within-ESU pairwise $F_{\mathrm{ST}}$ values; significant values are marked with an asterisk $(*)$, however, only values shown in bold are significant after sequential Bonferroni correction for multiple comparisons. Population $\mathrm{L}(N=1$, with one exclusive haplotype) was omitted from the matrix due to insufficient sample size; this population had recently declined due to disease and only one frog could be found.

\begin{tabular}{|c|c|c|c|c|c|c|c|c|c|c|c|c|c|c|c|}
\hline Pop. & $N$ & $h$ (excl.) & A & B & C & $\mathrm{D}$ & E & $\mathrm{F}$ & G & $\mathrm{H}$ & I & $\mathrm{J}$ & K & $\mathrm{M}$ & $\mathrm{N}$ \\
\hline A & 26 & | & & & & & & & & & & & & & \\
\hline B & 9 & 5 (5) & $0.689^{*}$ & & & & & & & & & & & & \\
\hline C & 9 & $6(5)$ & | $0.687^{*}$ & 0.063 & & & & & & & & & & & \\
\hline $\mathrm{D}$ & 10 & 2 (1) । & $0.704^{*}$ & $0.421^{*}$ & $0.642^{*}$ & & & & & & & & & & \\
\hline E & 10 & $2(2)$ & $0.711^{*}$ & $0.613^{*}$ & $0.763^{*}$ & $0.643^{*}$ & & & & & & & & & \\
\hline F & 6 & $5(3)$ & $0.206^{*}$ & $0.924^{*}$ & $0.927^{*}$ & $0.944^{*}$ & $0.951^{*}$ & & & & & & & & \\
\hline G & 9 & 7 (4) । & $0.118^{*}$ & $0.796^{*}$ & $0.793^{*}$ & $0.821^{*}$ & $0.829^{*}$ & 0.041 & & & & & & & \\
\hline $\mathrm{H}$ & 9 & $9(4)$ & $0.207^{*}$ & $0.906^{*}$ & $0.907^{*}$ & $0.923^{*}$ & $0.929^{*}$ & 0.071 & 0.009 & & & & & & \\
\hline I & 10 & $6(3)$ & $0.218^{*}$ & $0.929 *$ & $0.932^{*}$ & $0.945^{*}$ & $0.951^{*}$ & $0.132^{*}$ & 0.043 & -0.039 & & & & & \\
\hline J & 7 & 3 (3) & 0.215 & $0.953^{*}$ & $0.958^{*}$ & $0.970^{*}$ & $0.977^{*}$ & $0.269^{*}$ & $0.122^{*}$ & 0.082 & $0.131^{*}$ & & & & \\
\hline K & 9 & 4 (1) & $0.219^{*}$ & $0.957^{*}$ & $0.961^{*}$ & $0.972^{*}$ & $0.978^{*}$ & $0.223^{*}$ & $0.127^{*}$ & $0.094^{*}$ & $0.130^{*}$ & $0.275^{*}$ & & & \\
\hline M & 10 & $4(4)$ & $0.297^{*}$ & $0.925^{*}$ & $0.927^{*}$ & $0.940^{*}$ & $0.946^{*}$ & $0.367^{*}$ & $0.246^{*}$ & $0.299 *$ & $0.356^{*}$ & $0.411^{*}$ & 0.047 & & \\
\hline $\mathrm{N}$ & 8 & $4(2)$ & $0.271^{*}$ & $0.831^{*}$ & $0.829^{*}$ & $0.852^{*}$ & $0.861^{*}$ & $0.251^{*}$ & 0.091 & $0.251^{*}$ & $0.337^{*}$ & $0.380^{*}$ & $0.403^{*}$ & $0.438^{*}$ & \\
\hline $\mathrm{O}$ & 8 & 5 (3) & $0.360^{*}$ & $0.936^{*}$ & $0.939^{*}$ & $0.952^{*}$ & $0.959^{*}$ & $0.424^{*}$ & $0.218^{*}$ & $0.383^{*}$ & $0.510^{*}$ & $0.619^{*}$ & $0.637^{*}$ & $0.600^{*}$ & -0.042 \\
\hline Total & 141 & $60(49)$ & & & & & & & & & & & & & \\
\hline
\end{tabular}

distance was also significant if the taxa are analysed as representing a single species (Mantel: $r=0.56, P<0.005$ ), even after controlling for correlations associated with ESU membership (partial-Mantel: $r=0.37, P=0.005$ ). The general pattern of isolation by distance is also observed in the analyses of the separate species after correcting for ESU membership; while the pattern remains significant in $A$. zeteki when considered alone, the relationship is not significant in $A$. varius after controlling for correlations associated with ESU membership (partial-Mantel: $r=0.79$, $P=0.043$, and $r=0.25, P=0.062$, respectively).

Examining the genetic similarity of individuals from the same ESUs, compared to individuals from different ESUs, there is a significant relationship between genetic distance and ESU membership when all five ESUs are considered together (Mantel: $r=0.52, P<0.005$ ), even after the effect of geographic distance is removed (partial-Mantel: $r=0.29$, $P=0.007)$. In the analyses of the species separately, the correlation between genetic distance and ESU membership was only significant in A. varius (Mantel: $r=0.57, P<0.005$; partial-Mantel: $r=0.454, P=0.007$ with the effect of geographic distance removed, whereas in A. zeteki the correlation was Mantel: $r=0.363, P=0.099$ ).

\section{Phenotypic and environmental variation}

Analyses of the five ESUs detected significant differences in the means of each of the four morphological variables (SVL, \% black, z-yellow and z-black, Kruskal-Wallis: $\chi_{4}^{2} \geq 17.37, P \leq 0.002$ ). Pairwise comparisons to identify which ESUs differed significantly from each other revealed that the mean phenotypes did not differ among all the ESUs (Dunnett's C, Table 4). For example, the extent of black patterning on the dorsum (\% black) differed in $9 / 10$ pairwise comparisons and body size (SVL) differed in 8/10 comparisons. Surprisingly, the pairs of ESUs that did not show significant differences in these two phenotypic variables (ESUs 2 and 3 for the black trait, and ESUs 1 and 3 and ESUs 1 and 4 for SVL) are found in different mtDNA clades (one from $A$. zeteki clade and the other from $A$. varius clade, see Figs 1-2). These ESUs also exhibit significant differences in the other morphological variables, suggesting that the similarities in the extent of black patterning on the dorsum and body size are likely due to convergence.

Comparisons between the species also demonstrated a significant difference in two morphological variables, body size (SVL, Kruskal-Wallis: $\chi_{1}^{2}=8.85, P=0.005$ ) and extent of black patterning on the dorsum (\% black, KruskalWallis: $\chi_{1}^{2}=24.69, P<0.001$ ), but not in the other two morphological traits (z-yellow and z-black). Comparing the mean phenotypic variables among the ESUs in the separate species showed that ESUs in A. varius (ESUs 3-5, Fig. 1) differ in all four morphological variables (SVL, \% black, z-yellow, z-black, Kruskal-Wallis: $\chi_{2}^{2} \geq 14.27, P \leq$ 0.001) whereas the ESUs in A. zeteki (ESUs 1-2, Fig. 1) differ in all morphological variables (SVL, \% black, and z-yellow, Kruskal-Wallis: $\chi_{1}^{2} \geq 5.24, P \leq 0.022$ ) except black colouration (z-black, Kruskal-Wallis: $\chi_{2}^{2}=0.612, P=0.434$ ).

DFAs showed that the morphological variables (SVL, z-yellow and z-black colour values, and percentage black 
(a)

\begin{tabular}{lrllr}
\hline Source of variation & & & \% of total \\
& d.f. & Q-statistic* & variance & $P$ value \\
\hline Among 5 ESUs: (A-C)(D-E)(F-I)(J-M)(N-O) & 4 & $\Phi_{\mathrm{CT}}=0.465$ & 46.52 & 0.006 \\
Among populations within ESUs & 10 & $\Phi_{\mathrm{SC}}=0.498$ & 26.54 & $<0.001$ \\
Among individuals within a population & 126 & $\Phi_{\mathrm{ST}}=0.732$ & 26.85 & $<0.001$
\end{tabular}

${ }^{*} \mathrm{CT}$, variance among groups of populations, SC, variance among populations within groups, ST, variance among the individuals within a population.

(b)

\begin{tabular}{lrrrr}
\hline Source of variation & & & \% of total & \\
& d.f. & $\Phi$-statistic & variance & $P$ value \\
\hline Among species: (A-E)(F-O) & 1 & $\Phi_{\mathrm{CT}}=0.511$ & 51.05 & $<0.001$ \\
Among populations within species & 13 & $\Phi_{\mathrm{SC}}=0.566$ & 27.69 & $<0.001$ \\
Among individuals within a population & 126 & $\Phi_{\mathrm{ST}}=0.787$ & 21.25 & $<0.001$ \\
\hline
\end{tabular}

(c)

\begin{tabular}{lrlll}
\hline Source of variation & & & \% of total \\
& d.f. & $\Phi$-statistic & variance & $P$ value \\
\hline Among $A$. varius ESUs: (F-I)(J-M)(N-O) & 2 & $\Phi_{\mathrm{CT}}=0.241$ & 24.06 & $<0.001$ \\
Among populations within ESUs & 7 & $\Phi_{\mathrm{SC}}=0.096$ & 7.27 & $<0.001$ \\
Among individuals within a population & 68 & $\Phi_{\mathrm{ST}}=0.313$ & 68.67 & $<0.001$ \\
\hline
\end{tabular}

(d)

\begin{tabular}{lrrrr}
\hline & & & \% of total \\
Source of variation & d.f. & $\Phi$-statistic & variance & $P$ value \\
\hline Among A. zeteki ESUs: (A-C)(D-E) & 1 & $\Phi_{\mathrm{CT}}=0.110$ & 11.00 & 0.502 \\
Among populations within ESUs & 3 & $\Phi_{\mathrm{SC}}=0.696$ & 61.92 & $<0.001$ \\
Among individuals within a population & 58 & $\Phi_{\mathrm{ST}}=0.729$ & 27.08 & $<0.001$ \\
\hline
\end{tabular}

d.f., degree of freedom.

patterning, or \% black) were moderately successful in discriminating among the five proposed ESUs with 77.5\% of frogs being classed correctly (Table 5a). The majority of the misclassifications (33/42) involved frogs assigned to an ESU in the opposite species. The first canonical discriminant function (CDF), which explains $61.8 \%$ of the variance, is strongly influenced by the extent of black patterning on the dorsum (\% black) indicating the importance of this variable in discriminating among ESUs. Body size (SVL) was most strongly correlated with the second CDF (explaining $28 \%$ of the variance) and the colour values (z-yellow and z-black) were most strongly correlated with the third and fourth CDFs (together explaining $10.2 \%$ of the variance). The DFA based on the same morphological
Table 3 Four analyses of molecular variance based on different partitioning of variance across the five proposed ESUs (to accommodate taxonomic uncertainty; see Materials and methods for details): (a) examines the partitioning of variance across the five proposed ESUs and populations, treating all individuals as if they were sampled from a single species, (b) considers the contribution of the putative species designations, Atelopus varius and Atelopus zeteki, on patterns of variation, and lastly, in (c) and (d) the analysis of variance is conducted on the two species separately, where either only the three proposed $A$. varius ESUs (c) or the two proposed A. zeteki ESUs (d) are considered.

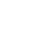

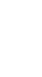




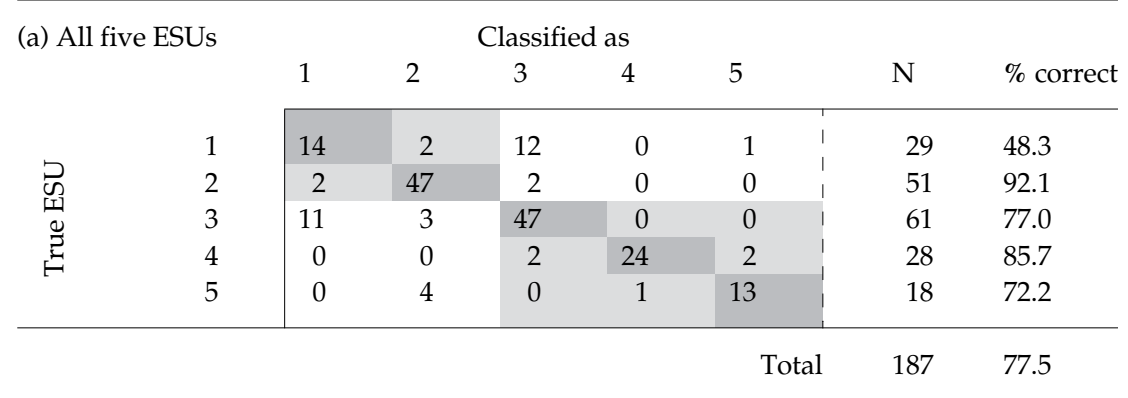

(b) A. varius vs. A. zeteki

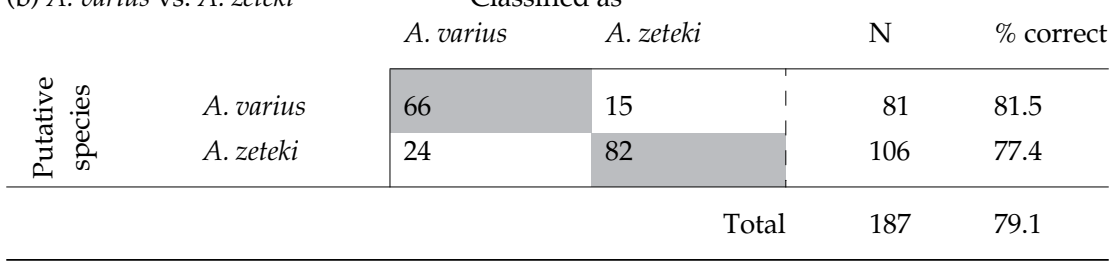

\begin{tabular}{|c|c|c|c|c|c|c|c|}
\hline \multirow{2}{*}{\multicolumn{2}{|c|}{ (c) A. varius ESUs }} & \multicolumn{4}{|c|}{ Classified as } & \multirow[b]{2}{*}{$\mathrm{N}$} & \multirow[b]{2}{*}{$\%$ correct } \\
\hline & & 3 & 4 & 5 & & & \\
\hline \multirow{3}{*}{ 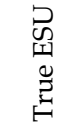 } & 3 & 58 & 0 & 3 & \multirow{2}{*}{$\begin{array}{l}1 \\
1\end{array}$} & 61 & 95.1 \\
\hline & 4 & 2 & 24 & 2 & & 28 & 85.7 \\
\hline & 5 & 1 & 1 & 16 & & 18 & 88.9 \\
\hline & & & & & & 107 & 91.6 \\
\hline
\end{tabular}

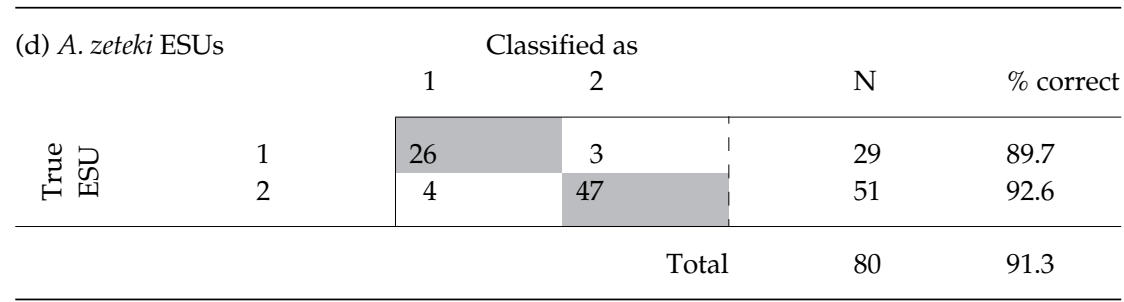

Table 5 Results of discriminant function analyses (DFAs) for models containing all four morphological variables (SVL, \% black, z-yellow and z-black), where correctly classified individuals are shown in dark grey along the diagonal, and $\mathrm{N}=$ number of individuals being classified for each category. The four tables represent analyses based on different partitionings (to accommodate taxonomic uncertainty, see Methods for details): (a) the five proposed ESUs, considering all golden frogs to be one taxon. (b) Atelopus varius vs. Atelopus zeteki, (c) the three proposed $A$. varius ESUs (A. zeteki individuals excluded), and (d) the two proposed $A$. zeteki ESUs (A. varius individuals excluded). In (b), light grey shading indicates incorrect ESU classifications within species. variables also successfully discriminated among the two species as $77.1 \%$ of frogs were classed correctly as either $A$. zeteki or A. varius (Table 5b). For this model, only one CDF was extracted, which explains $100 \%$ of the variance and is most strongly influenced by percentage black. Separate DFAs of the two species were each very successful at classifying individuals to their respective ESUs; $91.6 \%$ of frogs were classed correctly to ESUs within A. varius (Table 5c), and $91.3 \%$ of frogs were assigned correctly to ESUs in $A$. zeteki (Table 5d).

\section{Correlations among ecological, morphological, and genetic variation}

Pairwise comparisons across all populations showed that the magnitude of divergence in genetic, environmental and morphological variables differed (Table 6a). Only two of the morphological variables, SVL and percentage black, were correlated with environmental variables (Table 6a). The correlations between SVL and extent of black patterning with bioclim 1 and 2, respectively, and not genetic divergence, suggests that ecogenetic rather than phylogenetic factors account for these phenotypic population differences. Following these results, linear regressions revealed significant negative relationships between SVL and mean annual temperature $\left(r^{2}=0.282, P<0.001\right)$ and between percentage black and mean annual precipitation $\left(r^{2}=0.104, P<0.001\right)$.

The same relationship between SVL and mean annual temperature was also apparent in pairwise comparisons among populations of $A$. varius and A. zeteki considered separately, although the association was not as strong in A. zeteki (Tables $6 \mathrm{~b}$ and c). No significant correlations were 
Table 6 Correlations among genetic, morphological, and environmental variation showing the $P$ values trom the pairwise Mantel tests. Significant correlations are shown in bold and the corresponding $R$ values are also indicated.

(a) All populations together

\begin{tabular}{|c|c|c|c|c|c|c|c|}
\hline & SVL & z-yellow & z-black & $\%$ black & Bioclim 1 & Bioclim 2 & Bioclim 3 \\
\hline$F_{\mathrm{ST}}$ & 0.124 & 0.869 & 0.926 & 0.503 & 0.138 & 0.219 & 0.096 \\
\hline Bioclim 1 & $0.035^{*}$ & 0.427 & 0.464 & 0.051 & & & \\
\hline Bioclim 2 & 0.060 & 0.225 & 0.846 & $0.007^{* *}$ & & & \\
\hline Bioclim 3 & 0.226 & 0.051 & 0.976 & 0.957 & & & \\
\hline
\end{tabular}

${ }^{*} R=0.398,{ }^{* *} R=0.579$.

(b) Atelopus varius populations

\begin{tabular}{llllllll}
\hline & SVL & z-yellow & z-black & \% black & Bioclim 1 & Bioclim 2 & Bioclim 3 \\
\hline$F_{\text {ST }}$ & 0.059 & 0.258 & 0.736 & 0.261 & 0.091 & 0.095 & 0.805 \\
Bioclim 1 & $\mathbf{0 . 0 2 5 ^ { * }}$ & 0.510 & 0.713 & 0.708 & & \\
Bioclim 2 & 0.149 & 0.492 & 0.481 & 0.218 & & \\
Bioclim 3 & 0.948 & 0.377 & 0.339 & 0.459 & & \\
\hline
\end{tabular}

${ }^{*} R=0.724$.

(c) Atelopus zeteki populations

\begin{tabular}{|c|c|c|c|c|c|c|c|}
\hline & SVL & z-yellow & z-black & $\%$ black & Bioclim 1 & Bioclim 2 & Bioclim 3 \\
\hline$F_{\mathrm{ST}}$ & 0.381 & 0.421 & 0.401 & 0.776 & 0.881 & 0.453 & 0.846 \\
\hline Bioclim 1 & 0.072 & 0.310 & 0.921 & 0.820 & & & \\
\hline Bioclim 2 & 0.457 & 0.334 & 0.450 & 0.167 & & & \\
\hline Bioclim 3 & 0.170 & 0.062 & 0.673 & 0.314 & & & \\
\hline
\end{tabular}

SVL, snout-vent lengths.

found between genetic, morphological, or environmental variation among $A$. zeteki populations, which may reflect the smaller number of populations sampled (five vs. 10 populations in A. zeteki and A. varius, respectively). Linear regressions of SVL and mean annual temperature remained significant in each species (A. varius: $r^{2}=0.250, P<0.001$; A. zeteki: $\left.r^{2}=0.267, P<0.001\right)$; the regression of percentage black and mean annual precipitation was also significant in each species (A. varius: $r^{2}=0.397, P<0.001 ;$ A zeteki: $\left.r^{2}=0.150, P<0.001\right)$.

\section{Discussion}

The analyses confirm that there are not only significant genetic differences among the ESUs (Tables 2 and 3), but also significant divergence in morphological variables among the ESUs sufficient for discriminating ESU membership of individual frogs (Table 5). These conclusions were robust when each species was analysed separately, with the exception of Atelopus zeteki which lacked significant genetic differentiation among ESUs. This lack of genetic structure most likely reflects the large amount of variation within population $\mathrm{A}$, which contains highly divergent haplotypes characteristic of both species (Fig. 2). Despite the taxonomic uncertainty surrounding these endangered species (which was the rationale for conducting the analyses across the five ESUs together, as well as within each species separately), the species themselves are also clearly differentiated based on genetic (Fig. 2 and Table 3) and morphological data (Table 5). The results clearly support the taxonomic status of Atelopus varius and A. zeteki, and the proposed ESUs exhibit both morphological and genetic differences (as detected from significant differences in frequency of haplotypes; reciprocal monophyly was not observed, but it would also not be expected for recently diverged ESUs and species, as discussed below). However, a general lack of correspondence in the magnitude of divergence among variables suggests that multiple processes are contributing to the observed differentiation. Possible explanations for the patterns of correlated change (and lack thereof) among genetic, phenotypic, and environmental variables, and potential implications for the conservation of the endangered Panamanian golden frogs are discussed below. 
Patterns of differentiation and implications for the maintenance of variation in ESUs

Analyses of covariation among the genetic and phenotypic data revealed some complicated patterns across ESUs. The large amount of phenotypic differentiation observed between populations, ESUs, and species was apparent to varying degrees in the mtDNA phylogeny, the pairwise population $F_{\mathrm{ST}}$ values, and AMOVAs. This genetic structure, given the fine geographic scale of this study, suggests the frogs have remained fairly isolated. For example, among six populations of $A$. varius separated by less than $10 \mathrm{~km}$ (populations F-J, Fig. 1), there are 30 haplotypes represented, 17 of which are exclusive to a single population (Table 2). Moreover, significant isolation by distance is also apparent across populations, again suggesting the importance of limited gene flow in maintaining variation in the frogs. Nevertheless, when the effect of geographic distance was controlled (through the use of partial-correlation analyses), the ESUs remained genetically distinguishable (i.e. individuals from the same ESU were genetically more similar compared to individuals from different ESUs). This was true irrespective of whether all five ESUs were considered together or analysed separately in the two species $(P<0.05$, except for $A$. zeteki ESUs where fewer populations were compared and the relationship between ESU comembership and genetic similarity was nonsignificant, $P=0.099$ ). This again would support their long-term isolation, although it suggests that factors other than limited gene flow owing to the geographic distribution of populations may have contributed to the observed differentiation. The concordant boundaries of differentiation between the genetic and phenotypic data among ESUs and between species highlight the fact that patterns of differentiation across multiple characters have been strongly influenced by a common history. Yet the absence of a strong correspondence between the degree of divergence in genetic and morphological variables (Table 6) suggests that factors other than geographic constraints on gene flow contribute to the maintenance of variation in ESUs (see below).

\section{Processes underlying divergence as revealed by consideration of multiple characters}

In addition to avoiding the potential pitfalls involved with diagnosing conservation units based on neutral genetic data alone (Paetkau 1999; Gompert et al. 2006; Lorenzen et al. 2006), incorporating data from phenotypic traits is also integral if such units are to capture variation relevant to a species' ability to contend with various threats through adaptive change. Two implicit assumptions when ESUs are defined from patterns of phenotypic variation are that the variation (i) is largely heritable (as opposed to environmental), and (ii) is adaptive (rather than a consequence of drift or selection on a genetically correlated trait). Estimates of trait heritability require measurements of individuals with a known pedigree in a setting, such as a laboratory or a common garden, where environmental factors can be controlled. Explicit tests that individuals with the putatively adaptive trait exhibit greater survival or reproduction, compared with those lacking the trait, are then necessary to provide direct evidence for the adaptive significance of the character. Such explicit tests are often unrealistic for ESU studies as time, resources and access to animals are often limited. It is therefore no surprise that studies may rely on the untested assumption that phenotypic traits are both heritable and adaptive (e.g. Turgeon \& Bernatchez 2003).

Intraspecific phenotypic variation falls into two distinct classes: ecogenetic and phylogenetic (Thorpe et al. 1991; Rosso et al. 2004). Ecogenetic variation can be generated by selection in response to current or recent ecological factors, as well as by adaptive phenotypic plasticity or selection acting on a genetically correlated trait. In contrast, phylogenetic variation is generated by dispersal and vicariance events experienced by populations historically. However, the relative roles of ecogenetic and phylogenetic factors in generating variation among ESUs are not generally distinguished. Here we used an alternative to explicit tests of heritable adaptive variation that also potentially provides insight into whether such variation reflects ecogenetic and/or phylogenetic factors. Specifically, comparisons of character variation across multiple populations were used to determine whether there is an association between traits and putative selective factors, thereby indicating the potential relevance of the characters to adaptive divergence among ESUs. The significant associations detected between body size and colour patterning and environmental factors (Table 6) suggest that ESUs in the Panamanian golden frogs capture potentially adaptive variation (see details below). It is noteworthy that other anuran species also exhibit divergence in body size that co-varies with ecological factors, such as temperature, altitude, and precipitation (Berven 1982; Nevo 1971, 1973; Camp \& Marshall 2000). However, the general concordance of the ESU boundaries with both patterns of genetic and phenotypic differentiation (Tables 3,4, and 5), even if the magnitude of divergence in the multiple variables are not necessarily strongly correlated (Table 6), demonstrates that historical factors are also important in explaining golden frog variation.

Temperature and body size. In amphibians, body size (SVL) variation often reflects ecological differences among populations (reviewed in Ashton 2002). For golden frogs, body size and temperature are negatively correlated, meaning that smaller-bodied frogs are found at higher temperatures. This negative correlation has been found in 
a number of amphibian species and is considered to be a special case of Bergman's rule, as reviewed by Ashton (2002). While the proximate mechanism (i.e. selection or plasticity) behind this correlation has yet to be studied for golden frogs, other studies showing similar correlations between amphibian adult body size and temperature have attributed them to phenotypic plasticity (Camp \& Marshall 2000), selection (Nevo 1971, 1973), or both (Berven 1982) to avoid desiccation. Adult body size in amphibians depends on a number of factors which are likely subject to different levels of selection and degrees of phenotypic plasticity, including time and size at metamorphosis, growth rate pre- and post-maturity, age at maturity, and longevity (Smith 1987; Hemelaar 1988; Semlitsch et al. 1988; Moravec 1990; Augert \& Joly 1993; Friedl \& Klump 1997; Rosso et al. 2004). Capture-mark-recapture and skeletochronology studies are currently underway to determine whether or how these factors vary among the golden frog populations.

Colouration and genetic variation. Two colour variables (z-yellow and z-black) do not show strong support for either ecogenetic or phylogenetic forces in shaping their variation. In contrast, covariation in the extent of black patterning on the dorsum (\% black) with environmental differences but not with mtDNA sequence differences (Table 6) indicates that the character is affected by ecogenetic, rather than phylogenetic factors. This variable was correlated with precipitation-related climatic variables (see bioclim 2, Table 1) and a post hoc linear regression revealed that precipitation and percentage black are negatively correlated so that frogs have less black patterning in areas of high annual precipitation.

Black patterning on the dorsum is an adaptation or plastic response to prevent the harmful effects of ultraviolet light-B (UV-B) radiation, which for many organisms include immunosuppression and carcinogenic and mutagenic effects (Setlow 1974; Black \& Chan 1977; Jagger 1985). Melanin, the black pigment found in amphibian skin, has been demonstrated to reduce the effectiveness of UV-B penetration of amphibian eggs, larvae, and adults (reviewed in Licht \& Grant 1997). The amount of UV-B that reaches the Earth is affected by a number of factors, including ozone, sunspot activity, latitude and the angle of the sun, which are not likely to vary appreciably over the small geographic range of Panamanian golden frogs. Cloud cover, on the other hand, can vary over small geographic areas and dramatically affects the incidence of UV-B radiation (Bjorn 1989; Wellburn 1994). As areas of high mean annual precipitation undoubtedly experience more cloudy days than areas of low precipitation, the inverse relationship between black patterning and precipitation is consistent with the idea that dorsal black patterning is an adaptation for reducing the harmful effects of UV-B.
Support for the separate taxonomic status of A. varius and $\mathrm{A}$. zeteki

All the genetic analyses - pairwise population $F_{\mathrm{ST}}$ values, AMOVAs, and the high degree of exclusivity of haplotypes to populations - consistently indicate that there is significant structuring of variation across populations and ESUs. Partitioning populations and individuals by species also identified significant genetic differences between $A$. varius and A. zeteki; both species and ESU explained a greater amount of genetic variation than did the effect of population within the ESUs (Table 3), with the exception of $A$. zeteki, where genetic structuring is strong among populations within an ESU (likely due to the presence of the divergent ' $A$. varius haplotypes' in population A of A. zeteki, Table 3d, Fig. 2). In addition to significant genetic differentiation among species, DFAs based on multiple phenotypic differences provided accurate discrimination of the species, as well as the ESUs within each taxon (Table 5). This discriminatory ability was higher when $A$. varius and A. zeteki are considered to be distinct species, as opposed to treating them as a single taxon.

Nevertheless, A. varius and A. zeteki are not reciprocally monophyletic. The species' boundaries correspond generally to two strongly supported clades. However, three populations within the Rio Cocle del Norte drainage contain a mix of haplotypes from both clades (populations A, G, and $N)$. This pattern could have been generated by secondary contact with gene flow among formerly allopatric species, or alternatively, could reflect incomplete sorting of haplotypes among incipient taxa (Knowles 2004). Microsatellite markers are currently being developed to aid in distinguishing among these possibilities.

The lack of reciprocal monophyly in the mitochondrial gene tree itself is not necessarily inconsistent with the separate species status of $A$. varius and A. zeteki. Wellestablished population genetic theory raises significant concerns about the use of genetic thresholds like reciprocal monophyly to delimit species (Takahata \& Nei 1985; Hudson \& Coyne 2002; Hudson \& Turelli 2003; Moritz \& Cicero 2004; Matz \& Nielsen 2005; Knowles \& Carstens $2007 \mathrm{a}, \mathrm{b})$. For example, expectations of reciprocal monophyly derived from population genetic theory (Hudson 1992; Wakeley 2006) indicate that a substantial amount of time is required after the initial divergence of species before there will be a high probability of observing reciprocal monophyly at a sample of multiple loci (Hudson $\&$ Coyne 2002). Recently derived species will tend to go undiscovered under a reciprocal monophyly criterion since species' boundaries are not faithfully reflected in a gene tree until ancestral polymorphism has been fully sorted (e.g. Hickerson et al. 2006; Maddison \& Knowles 2006; Carstens \& Knowles 2007). 


\section{Conclusions}

This study demonstrates the utility of multiple character analyses, and more specifically tests for concordant variation among characters, in evaluating the support for proposed ESUs, especially in highly variable taxa such as Panamanian golden frogs. While many ESU studies include genetic data, only a few include ecological or morphological data. With the inclusion of such data, not only is it possible to evaluate whether ESUs are likely to preserve adaptive variation, but it also provides a framework to test for concordant variation among phenotypic and genetic variation that may give insights into the process(es) by which such variation has evolved. A general concordance between genetic and phenotypic differentiation among ESUs is evidence of the influence of historical factors on patterns of divergence, and supports the separate species status of $A$. varius and A. zeteki. The analyses also identified variation in body size and the extent of dorsal black patterning as putative adaptations to climatic factors affecting the likelihood of desiccation and the deleterious effects of UV-B radiation, respectively. Overall, the analyses of multiple characters and tests of character concordance provide strong evidence in support of the conservation of the two critically endangered species, as well as their constituent ESUs.

\section{Acknowledgements}

This research was supported by an NSF Graduate Research Fellowship and an NSF Doctoral Dissertation Improvement Grant (0608147) to C.L.R. in addition to fellowships from the Helen O. Brower Foundation, the University of Michigan's Rackham Graduate School, and the Society for the Study of Amphibians and Reptiles. Permission to research Atelopus varius and Atelopus zeteki, as well as to collect, import, and export tissue samples was provided by the Panamanian environmental authority (ANAM), the US Fish and Wildlife Service and the University of Michigan's Committee on the Use and Care of Animals (IACUC) to C.L.R. We are grateful to Tim Connallon, Joni Criswell, Edgardo Griffith, Wendy Grus, Tanya Hawley, Roberto Ibanez, Mark Iwen, Cesar Jaramillo, Nancy Karraker, Santiago Navas, Nick Quiring, Heidi Ross, Tracy Stetzinger, Scott Washkowiak, Lynn Zippel and Kevin Zippel for field assistance and assistance in acquiring tissue samples.

\section{References}

Ashton KG (2002) Do amphibians follow Bergman's rule? Canadian Journal of Zoology, 80, 708-716.

Augert D, Joly P (1993) Plasticity of age at maturity between two neighboring populations of the common frog (Rana temporaria). Canadian Journal of Zoology, 71, 26-33.

Avise JC (1989) A role for molecular genetics in the recognition and conservation of endangered species. Trends in Ecology $\mathcal{E}$ Evolution, 4, 274-278.

Berven KA (1982) The genetic basis of altitudinal variation in the wood frog Rana sylvatica. I. An experimental analysis of life history traits. Evolution, 36, 962-983.

Bjorn LO (1989) Computer programs for estimating ultraviolet radiation in daylight. In: Radiation Measurement in Photobiology (ed. Duffey BL), pp. 161-183. Academic Press, New York.

Black HS, Chan JT (1977) Experimental ultraviolet light carcinogenesis. Photochemistry and Photobiology, 26, 183-199.

Bohonak AJ (2002) IBD (isolation by distance): a program for analyses of isolation by distance. Journal of Heredity, 93, 153-154.

Bowen BW (1999) Preserving genes, species, or ecosystems? Healing the fractured foundations of conservation policy. Molecular Ecology, 8, S5-S10.

Camp CD, Marshall JL (2000) The role of thermal environment in determining the life history of a terrestrial salamander. Canadian Journal of Zoology, 78, 1702-1711.

Carstens BC, Knowles LL (2007) Estimating species phylogeny from gene-tree probabilities despite incomplete lineage sorting: an example from Melanoplus grasshoppers. Systematic Biology, $56,1-12$.

Cocroft RB, McDairmid RW, Jaslow AP, Ruiz-Carranza RM (1990) Vocalizations of eight species of Atelopus (Anura: Bufonidae) with comments on communication in the genus. Copeia, 1990, 631-643.

Crandall KA, Bininda-Emonds ORP, Mace GM, Wayne RK (2000) Considering evolutionary processes in conservation biology. Trends in Ecology \& Evolution, 15, 290-295.

Dimmick WW, Ghedotti MJ, Grose MJ, Maglia AM, Meinhardt DJ, Pennock DS (1999) The importance of systematic biology in defining units of conservation. Conservation Biology, 13, 653660.

Dizon AE, Lockyer C, Perrin WF, Demaster DP, Sisson J (1992) Rethinking the stock concept - a phylogeographic approach. Conservation Biology, 6, 24-36.

Dunn ER (1933) Amphibians and reptiles from El Valle de Anton, Panama. Occasional Papers of the Boston Society of Natural History, 8, 65-79.

Excoffier L, Smouse PE, Quattro JM (1992) Analysis of molecular variance inferred from metric distances among DNA haplotypes-application to human mitochondrial-DNA restriction data. Genetics, 131, 479-491.

Friedl TWP, Klump M (1997) Some aspects of population biology in the European treefrog, Hyla arborea. Herpetologica, 53, 321-330.

Friesen VL, Gonzalez JA, Cruz-Delgado F (2006) Population genetic structure and conservation of the Galapagos petrel (Pterodroma phaeopygia). Conservation Genetics, 7, 105-115.

Gharrett AJ, Mecklenburg CW, Seeb LW et al. (2006) Do genetically distinct rougheye rockfish sibling species differ phenotypically? Transactions of the American Fisheries Society, 135, 792-800.

Goebel AM, Donnelly JM, Atz ME (1999) PCR primers and amplification methods for 12s ribosomal DNA, the control region, cytochrome oxidase I, and cytochrome $b$ in bufonids and other frogs, and an overview of PCR primers which have amplified DNA in amphibians successfully. Molecular Phylogenetics and Evolution, 11, 163-199.

Gompert Z, Nice CC, Fordyce JA, Forister ML, Shapiro AM (2006) Identifying units for conservation using molecular systematics: the cautionary tale of the Karner blue butterfly. Molecular Ecology, 15, 1759-1768.

Grady JM, Quattro JM (1999) Using character concordance to define taxonomic and conservation units. Conservation Biology, 13, 1004-1007. 
Grant WS (1995) Multidisciplinary approaches for defining evolutionarily significant units for conservation. South African Journal of Science, 91, 65-67.

Green DM (2005) Designatable units for status assessment of endangered species. Conservation Biology, 19, 1813-1820.

Hasegawa M, Kishino H, Yano T-A (1985) Dating the human-ape splitting by a molecular clock of mitochondrial DNA. Journal of Molecular Evolution, 22, 160-174.

Hemelaar A (1988) Age, growth and other population characteristics of Bufo bufo from different latitudes and altitudes. Journal of Herpetology, 22, 369-388.

Hickerson MJ, Meyer CP, Moritz C (2006) DNA barcoding will often fail to discover new animal species over broad parameter space. Systematic Biology, 55, 729-739.

Hijmans RJ, Cemeron SE, Parra JL, Jones PG, Jarvis A (2005) Very high resolution interpolated climate surfaces for global land areas. International Journal of Climatology, 25, 1965-1978.

Holland BS, Dawson MN, Crow GL, Hofmann DK (2004) Global phylogeography of Cassiopea (Scyphozoa: Rhizostomeae): molecular evidence for cryptic species and multiple invasions of the Hawaiian Islands. Marine Biology, 145, 1119-1128.

Hood GM (2000) POPTOOLs: software for the analysis of ecological models. http://www.cse.csiro.au/poptools.

Hudson RR (1992) Gene trees, species trees and the segregation of ancestral alleles. Genetics, 131, 509-512.

Hudson RR, Coyne JA (2002) Mathematical consequences of the genealogical species concept. Evolution, 56, 1557-1565.

Hudson RR, Turelli M (2003) Stochasticity overrules the 'threetimes rule': genetic drift, genetic draft, and coalescence times for nuclear loci versus mitochondrial DNA. Evolution, 57, 182-190.

Huelsenbeck JP, Ronquist F (2001) MRBAYEs: Bayesian inference of phylogenetic trees. Bioinformatics, 17, 754-755.

IUCN, Conservation International, and NatureServe (2004) Global Amphibian Assessment. http://www.Globalamphibians.Org.

Jagger J (1985) Solar-UV Actions on Living Cells. Praeger, New York.

Knowles LL (2004) The burgeoning field of statistical phylogeography. Journal of Evolutionary Biology, 17, 1-10.

Knowles LL, Carstens BC (2007a) Inferring a populationdivergence model for statistical-phylogeographic tests in montane grasshoppers. Evolution, 61, 477-493.

Knowles LL, Carstens BC (2007b) Delimiting species without monophyletic gene trees. Systematic Biology, in press.

Legendre P, Legendre L (1998) Numerical Ecology. Elsevier, New York.

Licht LE, Grant KP (1997) The effects of ultraviolet radiation on the biology of amphibians. American Zoologist, 37, 137-145.

Lorenzen ED, Arctander P, Siegismund HR (2006) Regional genetic structuring and evolutionary history of the impala Aepyceros melampus. Journal of Heredity, 97, 119-132.

Lotters S (1996) The Neotropical Toad Genus Atelopus. Vences M, Glaw F. Verlags GbR, Koln, Germany.

Maddison WP, Knowles LL (2006) Inferring phylogeny despite incomplete lineage sorting. Systematic Biology, 55, 21-30.

Manly BFJ (2005) Multivariate Statistical Methods: A Primer. Chapman \& Hall/CRC, Boca Raton, Florida.

Matz MV, Nielsen R (2005) A likelihood ratio test for species membership based on DNA sequence data. Philosophical Transactions of the Royal Society of London. Series B, Biological Sciences, 360, 1969-1974.

McCartney MA, Acevedo J, Heredia C et al. (2003) Genetic mosaic in a marine species flock. Molecular Ecology, 12, 2963-2973.
Minin V, Abdo Z, Joyce P, Sullivan J (2003) Performance-based selection of likelihood models for phylogeny estimation. Systematic Biology, 42, 674-683.

Monsen KJ, Blouin MS (2003) Genetic structure in a montane ranid frog: restricted gene flow and nuclear-mitochondrial discordance. Molecular Ecology, 12, 3275-3286.

Moravec J (1990) Postmetamorphic growth in the European treefrog (Hyla arborea). Acta Universitatis Carolinae Biologica, 34, 359-370.

Moritz C (1994) Defining evolutionarily-significant-units for conservation. Trends in Ecology \& Evolution, 9, 373-375.

Moritz C (2002) Strategies to protect biological diversity and the evolutionary processes that sustain it. Systematic Biology, 51, 238-254.

Moritz C, Cicero C (2004) DNA barcoding: promise and pitfalls. Public Library of Science, Biology, 2, 1529-1531.

Moritz C, Schneider CJ, Wake DB (1992) Evolutionary relationships within the Ensatina eschscholtzii complex confirm the ring species interpretation. Systematic Biology, 41, 273-291.

Nakagawa S (2004) A farewell to Bonferroni: the problems of low statistical power and publication bias. Behavioral Ecology, 15, 1044-1045.

Nei M (1987) Molecular Evolutionary Genetics. Columbia University Press, New York.

Nevo E (1971) Climatic adaptation in size of the green toad (Bufo viridis). Israel Journal of Medical Science, 8, 1010.

Nevo E (1973) Adaptive variation in size of cricket frogs. Ecology, 54, 1271-1281.

Paetkau D (1999) Using genetics to identify intraspecific conservation units: a critique of current methods. Conservation Biology, 13, 1507-1509.

Palumbi SR, Martin A, Romano S, McMillan WO, Stice L, Grabowski G (1991) The Simple Fool's Guide to PCR, Version 2.0. Department of Zoology, University of Hawaii, Honolulu, HI 96882, privately published document compiled by S. Palumbi.

Rising JD, Avise JC (1993) Application of genealogical-concordance principles to the taxonomy and the evolutionary history of the Sharp-tailed sparrow (Amnodramus caudacutus). Auk, 110, 844856.

Roback PJ, Askins RA (2005) Judicious use of multiple hypothesis tests. Conservation Biology, 19, 261-267.

Rosso A, Castellano S, Giacoma C (2004) Ecogeographic analysis of morphological and life-history variation in the Italian treefrog. Evolutionary Ecology, 18, 303-321.

Ryan PG, Bloomer P (1999) The long-billed lark complex: a species mosaic in southwestern Africa. Auk, 116, 194-208.

Ryder OA (1986) Species conservation and systematics: the dilemma of subspecies. Trends in Ecology E Evolution, 1, 9-10.

Santos S, Hrbek T, Farias IP, Schneider H, Sampaio I (2006) Population genetic structuring of the king weakfish, Macrodon ancylodon (Sciaenidae), in Atlantic coastal waters of South America: deep genetic divergence without morphological change. Molecular Ecology, 15, 4361-4373.

Savage JM (1972) The harlequin frogs, genus Atelopus, of Costa Rica and western Panama. Herpetologica, 28, 77-94.

Semlitsch RD, Scott DE, Pechmann HK (1988) Time and size at metamorphosis related to adult fitness in Ambystoma talpoideum. Ecology, 69, 184-192.

Setlow RB (1974) The wavelengths in sunlight effective in producing skin cancer: a theoretical analysis. Proceedings of the National Academy of Sciences, USA, 71, 3363-3366. 
Shchneider S, Roessli D, Excoffier L (2000) ARLEQUIN Version 2.000: A Software for Population Genetics Data Analysis. Genetics and Biometry Laboratory, University of Geneva, Geneva, Switzerland.

Slatkin M (1993) Isolation by distance in equilibrium and nonequilibrium populations. Evolution, 47, 264-279.

Smith DC (1987) Adult recruitment in chorus frogs: effects of size and date at metamorphosis. Ecology, 68, 344-350.

Summers K, Bermingham E, Weigt L, McCafferty S (1997) Phenotypic and genetic divergence in three species of dart-poison frogs with contrasting parental behavior. Journal of Heredity, 88, 8-13.

Swofford DL (2002) PAUP*. Phylogenetic Analysis Using Parsimony (*and Other Methods), Version 4.0. Sinauer Associates, Sunderland, Massachusetts.

Takahata N, Nei M (1985) Gene genealogy and variance of interpopulational nucleotide differences. Genetics, 110, 325-344.

Taylor BL, Dizon AE (1999) First policy then science: why a management unit based solely on genetic criteria cannot work. Molecular Ecology, 8, S11-S16.

Taylor EB, McPhail JD (1999) Evolutionary history of an adaptive radiation in species pairs of threespine sticklebacks (Gasterosteus): insights from mitochondrial DNA. Biological Journal of the Linnean Society, 66, 271-291.

Thorpe RS, Brown RP, Malhotra A, Wuster W (1991) Geographic variation and population systematics: distinguishing between ecogenetics and phylogenetics. Bulletin of Zoology, 58, 329335.

Turgeon J, Bernatchez L (2003) Reticulate evolution and phenotypic diversity in North American ciscoes, Coregonus ssp. (Teleostei: Salmonidae): implications for the conservation of an evolutionary legacy. Conservation Genetics, 4, 67-81.

Villa J, Wilson LD, Johnson JD (1988) Middle American Herpetology - A Bibliographic Checklist. University of Missouri Press, Columbia, Missouri.

Vogler AP, Kinsley CB, Glueck SB, Hill JM, DeSalle R (1993) Using molecular and ecological data to diagnose endangered popu- lations of the puritan tiger beetle Cicindela puritana. Molecular Ecology, 2, 375-384.

Wakeley J (2006) Coalescent Theory: An Introduction. Roberts, Greenwood Village, Colorado.

Waples RS (1991) Pacific salmon, Oncorhynchus spp., and the definition of 'species' under the Endangered Species Act. Marine Fisheries Review, 53, 11-22.

Wellburn A (1994) Air Pollution and Climate Change: The Biology Impact. Wiley, New York.

Yotsu-Yamashita M, Kim YH, Dudley SC et al. (2004) The structure of zetekitoxin $\mathrm{AB}$, a saxitoxin analog from the Panamanian golden frog Atelopus zeteki: a potent sodium-channel blocker. Proceedings of the National Academy of Sciences, USA, 101, 4346-4351.

Zippel K (2002) Conserving the Panamanian golden frog: Proyecto Rana Dorada. Herpetological Review, 33, 11-12.

Zippel K, Ibanez DR, Lindquist ED, Richards CL, Jaramillo ACA, Griffith EJ (in press) Implicaciones en la conservacion de las ranas doradas de Panama, asociadas a su revision taxonomica. Herpetotropicos, $\mathbf{3}$, in press.

This study took place as part of my $\mathrm{PhD}$ dissertation at the University of Michigan which takes an integrative approach to the conservation of Panamanian golden frogs (Atelopus varius and Ateloppus zeteki). The overarching goal of my research is to understand the evolutionary processes which lead to population divergence and speciation and how those processes can be effectively preserved for endangered taxa. In doing this, my work integrates studies of molecular and phenotypic variation (including morphological, ecological, and behavioural aspects of phenotype). L. Lacey Knowles is interested in the processes of speciation and sexual selection, as well as phylogeography and evolutionary radiations, particularly as they pertain to montane grasshoppers of the genus Melanoplus inhabiting the sky islands of the northwestern United States. 